Copyright (C) 2003 IEEE. Reprinted from Engineering in Medicine and Biology Society, 2003. Proceedings of the 25th Annual International Conference of the IEEE , vol.1, no., pp. 646- 648 Vol.1, 17-21 Sept. 2003

This material is posted here with permission of the IEEE. Internal or personal use of this material is permitted. However, permission to reprint/republish this material for advertising or promotional purposes or for creating new collective works for resale or redistribution must be obtained from the IEEE by writing to pubs-permissions@ieee.org. By choosing to view this document, you agree to all provisions of the copyright laws protecting it. 


\title{
Three-Dimensional Automatic Volume Registration of Carotid MR Images
}

\author{
Baowei Fei ${ }^{1}$, Shaoxiong Zhang ${ }^{2}$, Olivier Savado ${ }^{1}$, Jasjit Suri ${ }^{1}$, \\ Jonathan S. Lewin ${ }^{2,1}$, David L. Wilson ${ }^{1,2}$ \\ 'Department of Biomedical Engineering, Case Western Reserve University, Cleveland, OH, USA \\ ${ }^{2}$ Department of Radiology, University Hospitals of Cleveland, Cleveland, OH, USA
}

\begin{abstract}
We created an automatic three-dimensional registration algorithm for magnetic resonance images of carotid vessels. Potential applications include atherosclerotic plaque characterization and plaque burden quantification vector-based segmentation using dark blood MR images having multiple contrast weightings (proton density (PD), T1, and T2). Another application is measurement of disease progression and regression with drug trials. We used mutual information registration algorithm to compensate movements between image acquisitions. $\mathrm{PD}, \mathrm{T1}$, and $\mathrm{T} 2$ images were acquired from patients and volunteers and then matched for image analysis. Visualization methods such as contour overlap showed that vessels well aligned after registration. Distance measurements from the landmarks indicated that the registration method worked well with an error of $1.09 \pm 0.42 \mathrm{~mm}$.
\end{abstract}

Keywords-Image registration, dark blood MR image, carotid plaque classification

\section{INTRODUCTION}

Atherosclerotic disease of the carotid artery is the leading cause of stroke [1]. MRI has emerged as a potential leading in vivo imaging modality for atherosclerotic plaque characterization [2][3]. Black blood high-resolution MRI techniques with multiple contrast weightings (proton density (PD), T1, and T2) have been shown to be useful for atherosclerotic plaque characterization and plaque burden assessment [4].

Quantification during disease progression and after therapeutic intervention may improve our knowledge of the natural history of the disease and lead to improved therapeutic strategies. However, the spatial location mismatching of different scans from serial examination will impair the accuracy of quantified analysis of atherosclerotic disease using MRI. Image registration has the potential to improve the quantification and characterization [5]. Combination of multiple images such as proton density (PD), T1, and T2 could verify lesions and provide more information for diagnosis and treatment. Further, registration of serial examinations can follow up the progression and regression of diseases [6]. In this study, we perform registration. experiments for $\mathrm{MR}$ images from patients with carotid stenosis and volunteers.

\section{METHODOLOGY}

\section{A. Registration Algorithm}

We are investigating voxel-based methods for automatic three-dimensional (3D) registration because they do not depend on possibly inaccurate image segmentation and because they can be extended to non-rigid registration [7][9].

We used normalized mutual information (NMI) as the similarity measures in our registration because they are robust and suitable for multi-modality image registration [7]. Suppose one image $R$ is the reference, and the other $F$ is floating. NMI is given by the following equation [7].

where

$$
N M I(R, F)=\frac{2 M I(R, F)}{H(R)+H(F)}
$$

$$
\begin{aligned}
& H(R)=-\sum_{r} p_{R}(r) \log p_{R}(r), H(F)=-\sum_{f} p_{F}(f) \log p_{F}(f) \\
& M I(R, F)=\sum_{r, f} p_{R F}(r, f) \log \frac{p_{R F}(r, f)}{p_{R}(r) \cdot p_{F}(f)} .
\end{aligned}
$$

The joint probability $p_{R F}(r, f)$ and the marginal probabilities $p_{R}(r)$ of the reference image and $p_{F}(f)$ of the floating image, can be estimated from the normalized joint intensity histograms. When two images are geometrically aligned, NMI is maximal.

We used rigid body transformation (three translations and three angles) and trilinear interpolation. For optimization, we use the downhill simplex method of Nelder and Mead [10]. Optimization of alignment ends either when the maximum number of NMI calculations is reached (typically 500) or the fractional change in NMI is smaller than a tolerance (typically 0.0001). Our very first initial guesses are all zeros for the 3 displacements and 3 angles.

\section{B. Image Acquisitions}

All MR scans were conducted on a $1.5 \mathrm{~T}$ system (Magnetom Sonata, Siemens Medical Solutions, Erlangen, Germany) with a custom-built phased array coil to improve the local image signal-to-noise ratio. Patients were positioned supine on the scanner table. After axial, sagittal and coronal localizer images were acquired; a set of double oblique localizer images was then acquired to monitor the phased array coil position and to roughly identify the carotid 
artery bifurcation. A transverse three-dimensional (3D) multiple overlapping thin slab angiography (MOTSA) sequence with TR/TE/flip/partition thickness of $20 \mathrm{~ms} /$ $3.4 \mathrm{~ms} / 25 \% 1 \mathrm{~mm}$, was used to locate the exact level of the carotid bifurcation. Dark blood images were then obtained using ECG-triggered double inversion recovery (DIR) turbo spin echo sequences. The imaging parameters (TR/TE/TI/NSA/thickness/FOV) were as follows: 1R$\mathrm{R} / 7.1 \mathrm{~ms} / 500 \mathrm{~ms} / 2 / 3 \mathrm{~mm} / 13 \mathrm{~cm}$ (T1), $2 \mathrm{R}-\mathrm{R} / 7.1 \mathrm{~ms} / 600 \mathrm{~ms} / 2 /$ $3 \mathrm{~mm} / 13 \mathrm{~cm}$ (PD), $2 \mathrm{R}-\mathrm{R} / 68 \mathrm{~ms} / 600 \mathrm{~ms} / 2 / 3 \mathrm{~mm} / 13 \mathrm{~cm}$ (T2). Fat saturation was applied for all dark blood images. The in plane resolution was $0.51 \times 0.51 \mathrm{~mm}^{2}$. We acquired $\mathrm{PD}, \mathrm{T} 1$, and $\mathrm{T} 2$ images from one patient $\mathrm{P} 1$ and two volunteers $\mathrm{S} 1$ and $\mathrm{S} 2$.

\section{Registration Experiments}

We have nine pairs of volumes for registration experiments. For each subject, we have PD, T1 and T2 image volumes. We used PD as the reference image and we registered $\mathrm{T} 1$ and $\mathrm{T} 2$ with $\mathrm{PD}$. We also matched $\mathrm{T} 2$ with $\mathrm{T} 1$ to test the registration algorithm. There are three registration experiments for each subject. We performed two registration trials for volunteers S1 and S2. In total, we conducted 15 registration experiments, three for the patient $\mathrm{P} 1$ and three for each of the volunteers S1 and S2.

\section{Registration Evaluation}

We used visual inspections to evaluate the registration. We used RegViz, a program written in IDL (Interactive Data Language, Research System Inc., Boulder, CO) and created in our laboratory for visualizing and analyzing registered image volumes. First, we manually segmented carotid vessel walls in image slices and copied them to corresponding slices. This enabled visual determination of the overlap of vessel walls over the entire volume. Second, color overlay displays were used to evaluate overlap of structures. One image was rendered in gray and the other in the "hot-iron" color scheme available in IDL. To visualize potential differences, it was quite useful to interactively change the contribution of each image using the transparency scale. Third, we used a sector display, which divided the reference and registered images into rectangular sectors and created an output image by alternating sectors from the two input images. Even subtle shifts of edges would be clearly seen

We evaluated registration of the vessels by measuring the displacement of the landmarks following registration. We used the left and right bifurcations as the landmarks. To measure displacements, we navigated transverse, coronal, and sagittal MR images slice-by-slice to search bifurcations in both reference and registered volumes. Using consistent rules and magnified images, a radiologist used a cursor to identify unique features on both images. The 3D locations for each landmark were recorded and 3D distance between corresponding landmarks is computed.

\section{RESULTS}

\section{A. Visual Inspection}

We determined the quality of vessel registration by visually examining all image slices of registered volume pairs using one or more of the methods found in RegViz. A typical example is shown in Fig. 1 where the contour overlap is excellent and probably within the manual segmentation error. Other transverse images were also well aligned indicating that the registration was successful in three dimensions. Other visual inspection techniques such as color overlay and sector display also demonstrate excellent registration.

\section{B. Assessments of Bifurcations}

Following registration, we measured $3 \mathrm{D}$ distances between corresponding bifurcations. Registration results are shown in Table I. The average error across the three subjects with 15 registration experiments is only $1.09 \pm 0.42 \mathrm{~mm}$. The isotropic voxel size of the volumes is $0.51 \mathrm{~mm}$. The measured error reported for NMI registration is probably overestimated due to landmark location error.

TABLE I

DISPLACEMENT OF BIFURCATIONS MEAN (STANDARD DEVIATION) IN mm

\begin{tabular}{llll}
\hline & PD-T1 & PD-T2 & T1-T2 \\
\hline Left bifurcation & $1.04(0.63)$ & $1.49(0.56)$ & $1.02(0.27)$ \\
\hline Right bifurcation & $0.97(0.34)$ & $1.04(0.24)$ & $0.98(0.31)$ \\
\hline
\end{tabular}

\section{Implementation Issues}

The algorithm was quite robust for nine volume pairs in this study. Each call to the Simplex optimization resulted in 120 to 250 NMI evaluations before the tolerance $(0.0001)$ was reached. The time for a single registration, typically 5 minutes for the volumes with $256 \times 256 \times 59$-voxels on a Pentium IV, $1.8 \mathrm{GHz}$ CPU, with 1 Gbytes of memory, could probably be greatly improved with optimized $\mathrm{C}$ code rather than IDL.

\section{DISCUSSION \& CONCLUSION}

We have developed an automatic volume registration algorithm for multiple contrast weighted MR images of carotid vessels. Our internal measures showed the registration is quite robust and accurate. It will probably be a useful tool for many applications of interest in vascular imaging. 


\section{ACKNOWLEDGMENT}

The algorithm developed in this research was supported by DOD grant DAMD17-02-1-0230 to Baowei Fei and NIH grant R01-CA84433-01 to David L. Wilson.

\section{REFERENCES}

[1] G.W.Petty, R.D.Brown, J.P.Whisnant, J.D.Sicks, W.M.O'Fallon and D.O.Wiebers, "Ischemic stroke subtypes - A population-based study of functional outcome, survival, and recurrence," Stroke, vol. 31, pp. 1062-1068, 2000

[2] Z.A.Fayad and V.Fuster, "Clinical imaging of the high-risk or vulnerable atherosclerotic plaque," Circulation Research, vol. 89, pp. 305-316, 2001

[3] C.Yuan, L.M.Mitsumori, K.W.Beach, and K.R.Maravilla, "Carotid atherosclerotic plaque: Noninvasive MR characterization and identification of vulnerable lesions," Radiology, vol. 221, pp. 285$299,2001$.

[4] S.X.Zhang, T.S.Hatsukami, N.L.Polissar, C.Han, and C.Yuan, "Comparison of carotid vessel wall area measurements using three different contrast-weighted black blood MR imaging techniques," Magnetic Resonance Imaging, vol. 19, pp. 795-802, 2001.

[5] V.L.Stevenson, S.M.Smith, P.M.Matthews, D.H.Miller, and A.J.Thompson, "Monitoring disease activity and progression in primary progressive rnultiple sclerosis using MRI: sub-voxel registration to identify lesion changes and to detect cerebral atrophy," Journal of Neurology, vol. 249, pp. 171-177, 2002.

[6] D.Rey, G.Subsol, H.Delingette, and N.Ayache, "Automatic detection and segmentation of evolving processes in 3D medical images: Application to multiple sclerosis," Medical Image Analysis, vol. 6, pp. 163-179, 2002.

[7] F.Maes, A.Collignon, D. Vandermeulen, G.Marchal, and P.Suetens, "Multimodality image registration by maximization of mutual information," IEEE Trinsactions on Medical Imaging, vol. 16, pp. 187-198, 1997.

[8] B.W.Fei, C.Kemper, and D.L.Wilson, "A comparative study of warping and rigid body registration for the prostate and pelvic MR volumes," Computerized Medical Imaging and Graphics, vol. 27, pp. 267-281, 2003.

[9] B.W.Fei, A.Wheaton, Z.Lee, J.L.Duerk, and D.L.Wilson, "Automatic MR volume registration and its evaluation for the pelvis and prostate," Pinysics in Medicine and Biology, vol. 47, pp. 823-838, 2002.

[10] J.Nelder and R.A.Mead, "A simplex method for function minimization," Compuier Journal, vol. 7, pp. 308-313, 1965.
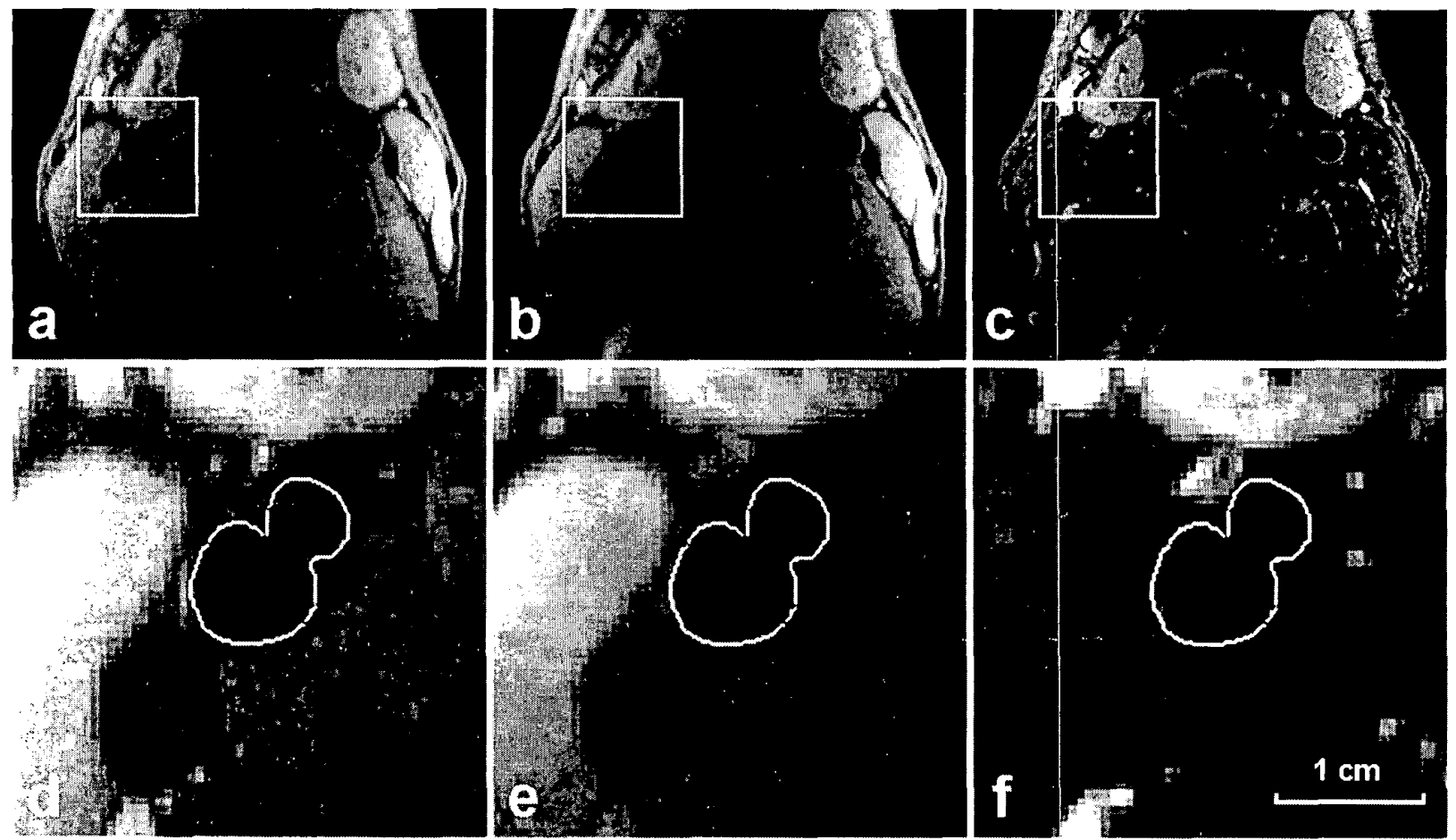

Fig. I. MR images of carotid vessels. The top images from left to right are proton density (PD), $\mathrm{T} 1$, and $\mathrm{T} 2$ images, respectively. ${ }^{\prime}$ Both $\mathrm{T} 1$ and $\mathrm{T} 2$ images were registered with the PD image. The rectangular regions are magnified and displayed at the bottom. The carotid vessel walls were manually segmented from the PD image (left) and copied to the T1 (center) and T2 images (right). The contour overlaps at the bottom shows that the vessel boundaries from PD well aligned with those in $\mathrm{T} 1$ and $\mathrm{T} 2$ images. Images are from Volunteer $\mathrm{S} 1$ 